\title{
The Wnt5a/Ror2 pathway is associated with determination of the differentiation fate of bone marrow mesenchymal stem cells in vascular calcification
}

\author{
HUAPING XIN, FANG XIN, SHAOQIONG ZHOU and SIMING GUAN \\ Department of Geriatrics, Union Hospital, Huazhong University of Science and Technology, Wuhan, P.R. China
}

Received November 1, 2012; Accepted December 17, 2012

DOI: $10.3892 /$ ijmm.2013.1242

\begin{abstract}
Accumulating evidence have demonstrated that mesenchymal stem cells (MSCs) are involved in the initiation and progression of various vascular diseases. Canonical Wnt signaling controls the fate of MSCs, and plays an important role in vascular calcification. However, vascular calcification can be inhibited by the non-canonical Wnt signaling pathway Wnt5a/ Ror2. This study aimed to investigate whether the Wnt5a/Ror2 pathway is associated with determination of the differentiation fate of MSCs in vascular calcification. Direct co-cultures were established by seeding smooth muscle cells (SMCs) or calcified SMCs and MSCs together at ratios of SMCs or calcified SMCs $15 \times 10^{4}$; SMCs or calcified SMCs $5 \times 10^{4}$ : MSCs $10 \times 10^{4}$, SMCs or calcified SMCs $10 \times 10^{4}$ : MSCs $5 \times 10^{4}$. Osteosynthesisinducing medium (OS) was added to the culture medium in the groups of MSCs with non-calcified SMCs. Cells were cultured for nine days. Osteoblastic differentiation was evaluated by cell morphology and the activity of alkaline phosphatase (ALP) in cell lysates and ALP staining. Furthermore, we investigated the inhibition of Wnt signaling, and observed that the members of the non-canonical signaling pathway Wnt5a/Ror2 were expressed in each group. Additionally, MSCs cultured in culture media with OS did not differentiate into an osteoblast phenotype when in direct contact with non-calcified SMCs, irrespective of the number of MSCs. However, an osteoblast phenotype was observed when MSCs were cultured in media without OS differentiation towards direct contact with calcified SMCs, and the levels of osteoblastic markers had a direct correlation with the number of MSCs. Of note, the Wnt5a protein was associated with the levels of calcification, thus, although rarely detected in non-calcification, Ror 2 mRNA in
\end{abstract}

Correspondence to: Professor Siming Guan, Department of Geriatrics, Union Hospital, Huazhong University of Science and Technology, 1277 Jiefang Avenue, Wuhan 430000, P.R. China E-mail: smguan16@yahoo.com

Key words: Wnt5a/Ror2 pathway, differentiation, bone marrow mesenchymal stem cells, vascular calcification the non-calcified groups was significantly higher compared to that in the calcified groups. Therefore, the Wnt5a/Ror2 pathway is associated with determination of the differentiation fate of bone marrow mesenchymal stem cells in vascular calcification.

\section{Introduction}

Vascular calcification affects our aging and dysmetabolic population (1). Vascular calcification is known to be an actively regulated osteogenic processes $(2,3)$. An important step of osteogenic processes is the osteochondrocytic differentiation of bone marrow (BM)-derived mesenchymal stem cells (MSCs) (4). MSCs can be isolated from bone marrow, harbor expansion potential, and differentiate into mesenhymal cells, such as osteoblasts and chondrocytes (5), as well as into nonmesenchymal cells, such as vascular cells $(4,6,7)$. Moreover, MSCs can enter the circulation and migrate into vascular and other connective tissues (8), especially at sites of injury and in tissue transplant grafts (9-11).

MSCs are of increasing interest for future therapeutic exploitation in the treatment of cardiovascular diseases in the setting of allogeneic hematopoietic stem cell transplantations (12). Although it is attractive to consider that growth of MSC in injured vascular tissue may simply regenerate normal vascular tissue, it is also possible for these cells to produce ectopic tissues, such as those observed in advanced atherosclerotic calcification (13). MSC are involved in the initiation and progression of various vascular diseases (10). Notably, Wnt-signaling controls the fate of MSCs, and plays an important role in vascular calcification (4). MSCs also contribute to the regulation of osteogenic mineralization during development and disease and are therefore important in vascular calcification $(14,15)$. However, vascular calcification can be inhibited by the non-canonical Wnt signaling pathway, Wnt5a/Ror2. Therefore, it is important to determine whether this pathway is associated with determination of the differentiation fate of MSCs in vascular calcification, since few studies are currently available regarding this novel method (17). In this study, we used an in vitro cell-cell co-culturing system to observe whether MSCs directly interact with vascular calcification and investigate the Wnt5a/Ror2 pathway during the process. 


\section{Materials and methods}

Rat bone-marrow-derived MSCs. The isolation and culture of male MSCs was performed as described in a previous study (17). MSCs from the rats were allowed to attach to culture flasks. After two days, non-attached cells were washed away and the cells on flasks were cultured for 1-2 weeks until near-confluence. MSCs were cultured in DMEM, supplemented with $10 \%$ heat-inactivated FBS (both from Invitrogen Life Technologies, Carlsbad, CA, USA), $100 \mathrm{U} / \mathrm{ml}$ penicillin and $100 \mathrm{Ag} / \mathrm{ml}$ streptomycin at $37^{\circ} \mathrm{C}$ in $5 \% \mathrm{CO}_{2}$ and $95 \%$ air using trypsin-EDTA solution (Invitrogen Life Technologies) to digest and detach MSCs. MSCs were used at passage three.

Rat aortic SMCs. Rat aortic SMCs (A-10, ATCC) were grown in low-glucose DMEM. Osteosynthesis-inducing medium (OS), used only in one osteoblastic differentiation assay, contained the above described medium with $0.1 \mu \mathrm{M}$ dexamethasone, $10 \mathrm{mM}$ sodium $\beta$-glycerol-phosphate and $0.05 \mathrm{mM}$ ascorbic acid-2-phosphate (Sigma-Aldrich, St. Louis, MO, USA). The culture medium was removed and replaced with fresh medium three times a week. SMCs were cultured for 21 days after the formation of calcified nodules.

Co-culture conditions. Direct co-cultures were established by seeding smooth muscle cells (SMCs) or calcified SMCs and MSCs together at ratios of SMCs or calcified SMCs $15 \times 10^{4}$; SMCs or calcified SMCs $5 \times 10^{4}$ : MSCs $10 \times 10^{4}$, SMCs or calcified SMCs 10x104: MSCs $5 \times 10^{4} / 1.7 \mathrm{~cm}^{2}$ into six-pore plates. OS was added in the culture medium in the groups of MSCs with non-calcified SMCs. Cells were cultured in low-glucose DMEM supplemented with $10 \% \mathrm{FBS}$ at $37^{\circ} \mathrm{C}$ in a $5 \% \mathrm{CO}_{2}$ incubator for 9 days. The medium was changed every 3 days. Each co-culture experiment was performed three times to validate the results. Direct co-cultures were established by seeding together two different cell types at three distinct cell density ratios and culturing the cells for 9 days. Osteoblastic differentiation was then evaluated by cell morphology, the activity of alkaline phosphatase (ALP) in cell lysates and ALP staining. Cell proliferation was evaluated by protein content. Additionally the inhibition of Wnt-signaling was investigated to determine whether the non-canonical signaling pathway, Wnt5a/Ror2, was expressed in each group.

Flow cytometry. Passage three MSCs were trypsinized, washed with PBS and incubated with fluorescein isothiocyanator phycoerythrin-conjugated monoclonal antibodies (BioLegend) specifically for CD29, CD90, CD45, CD11b or PBS in $4^{\circ} \mathrm{C}$ for $30 \mathrm{~min}$. Analysis was performed by flow cytometry (Becton-Dickinson, Franklin Lakes, NJ, USA), using CellQuest software.

von Kossa staining. For von Kossa staining, cells were fixed with paraformaldehyde (Sigma-Aldrich) at $4^{\circ} \mathrm{C}$ for $45 \mathrm{~min}$. The fixed cells were incubated in 5\% silver nitrate for $30 \mathrm{~min}$ in ultraviolet, and air-dried color development (black) was complete. Calcification was observed under light microscopy.

ALP staining. For alkaline phosphatase (ALP) activity analysis, cells were fixed with $4 \%$ paraformaldehyde (Sigma-Aldrich) for
15 min at room temperature, and then stained with the BCIP/ NBT Phosphatase Substrate System (KLP, Gaithersburg, MD, USA) following the manufacturer's instructions and observed under light microscopy.

ALP activity and protein content. ALP activity was determined from all the samples after being cultured for 9 days. Samples were extracted into an assay buffer containing $50 \mathrm{mM}$ Tris- $\mathrm{HCl}, 0.1 \%$ Triton $\mathrm{X}-100$ and $0.9 \% \mathrm{NaCl}$ (pH 7.6) and the lysate samples were frozen. Lysate samples were then thawed and enzyme activity was determined in duplicate using 0.1 M 4-p-nitrophenylphosphate as a substrate (Jiancheng, Nanjing, China). Absorbance was read at $492 \mathrm{~nm}$ in a plate reader (Bio-Rad, Hercules, CA, USA). The total protein contents were determined by Bio-Rad Protein Assay (Bio-Rad).

Ror2 mRNA expression. Total RNA was isolated from the samples using TRIzol reagent according to the manufacturer's instructions (Invitrogen) and reverse transcribed into cDNA using a Toyobo reverse transcription kit (Toyobo). The real-time quantitative PCR was carried out with the ABI PRISM 7900 sequence detector system (Applied Biosystems) according to the manufacturer's instructions. GAPDH was used as an endogenous control. PCR reaction mixture contained SYBR-Green I (Takara), cDNA, and the primers. Primer sequences used for real-time quantitative PCR were: Ror2, upstream: 5'-ATCCAAGACCTGGACACAACAGA-3' and downstream: 5'-GAACCCCAGTGGCAGTGATG-3'. Relative gene expression level (the amount of target, normalized to the endogenous, control gene) was calculated using the comparative $\mathrm{Ct}$ method formula $2^{-\Delta \Delta \mathrm{Ct}}$.

Western blot analysis. Cells were incubated with NET buffer (50 mM Tris, $5 \mathrm{mM}$ EDTA, $150 \mathrm{mM} \mathrm{NaCl}, 1 \mathrm{mM}$ PMSF, $20 \mathrm{mM}$ NEM, $1 \%$ NP 40, pH 7.4). Total protein was quantified using a BCA protein assay kit (Pierce-Perbio Science, Tattenhall, UK). The primary antibody used was anti-Wnt5a [rabbit polyclonal anti-Wnt5a (ab72583); Abcam, Cambridge, MA, USA] at a dilution of 1:1,000. For the normalization of protein loading, anti-GAPDH (cat. no. KC-MM-1302) was used at a dilution of 1:10,000. Equivalent amounts of protein were resolved by SDS-PAGE electrophoresis. Proteins were transferred onto polyvinylidene difluoride (PVDF) membranes, which were blocked in 5\% BSA and incubated with the relevant antibody. Western blotting was carried out using the chemiluminescence detection system (Bio-Rad).

Statistical analysis. Results were shown as the mean \pm SEM. The significance of differences was estimated by ANOVA followed by Student-Newmann-Keuls multiple comparison tests. $\mathrm{P}<0.05$ was considered statistically significant. Statistical analyses were performed using SPSS software (version 11.0; SPSS Inc., Chicago, IL, USA).

\section{Results}

Cultured passage three MSC. Passage three rat MSCs were positive at $\mathrm{CD} 29$ and $\mathrm{CD} 90$, but negative at $\mathrm{CD} 45$ and $\mathrm{CD} 1 \mathrm{~b}$ and different from hemopoietic stem cells (18) (Fig. 1). 
A

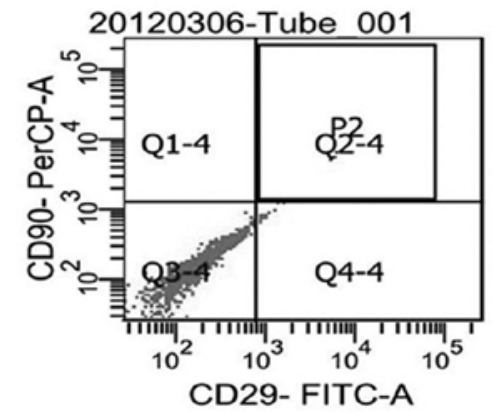

B

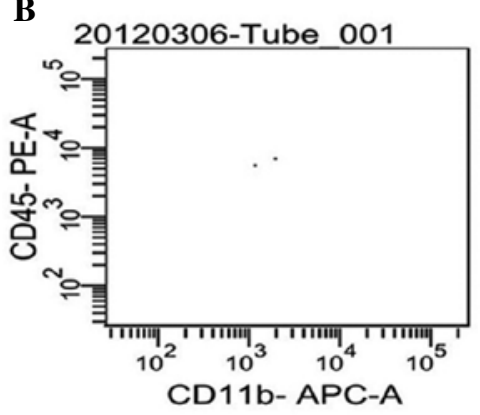

Figure 1. MSC cell-surface antigen analysis. (A) CD29 and CD90 expression was high at $\sim 99.1$ and $\sim 96 \%$, respectively, whereas the expression of (B) CD45 and CD11b was low at $\sim 0.6 \%$, respectively.
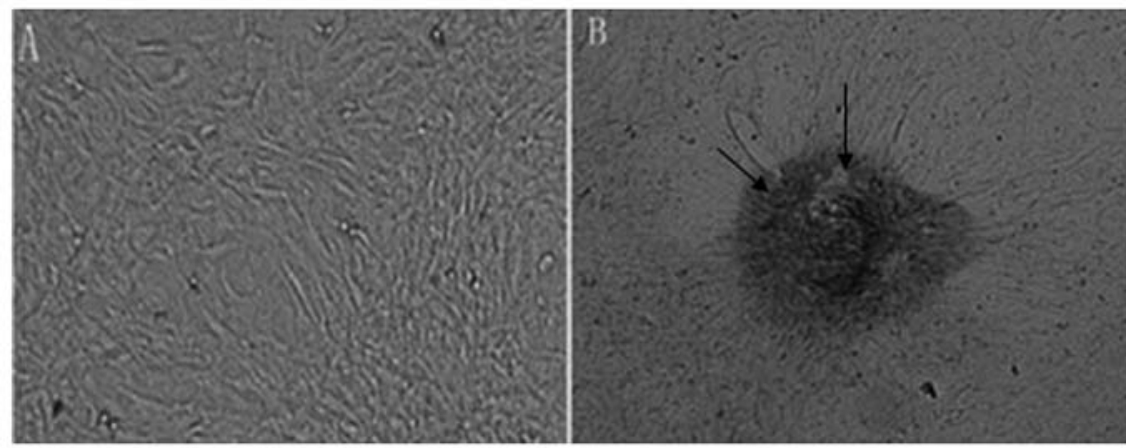

Figure 2. von Kossa staining showing SMCs cultured in medium with or without OS for 21 days. Representative photographs of von Kossa staining of SMCs cultured in media without OS. (A) No calcification is evident, (B, black arrowhead) black calcified nodules in SMCs cultured in media with OS. Original magnification, $\mathrm{x} 100$.

SMCs were cultured in medium with or without OS. SMCs were cultured in medium with or without OS for 21 days. Representative photographs of von Kossa staining are shown in Fig. 2. No calcification was detected in SMCs cultured without OS (Fig. 2A), whereas there were strongly positively stained black calcified nodules in SMCs cultured with OS (Fig. 2B, black arrowhead).

MSCs cultured in culture media with OS did not differentiate into an osteoblast phenotype when in direct contact with non-calcified SMCs, irrespective of the number of MSC. To determine whether non-calcified or calcified SMCs affected osteoblastic differentiation of co-cultured MSCs, ALP activity and ALP staining were quantified. A previous study demonstrated that OS significantly increased ALP activity when MSCs were cultured in the plastic culture flask (19). However, OS did not increase ALP activity when the cells were cultured in non-calcified SMCs. The results showed that the levels of all measured osteoblastic markers remained the same in the non-calcified SMCs directly co-cultured with MSCs at different cell ratios, ALP staining was negative, and there was no difference of ALP activity between the SMC15 (S15), SMC5:MSC10 (S5M10 + OS), and SMC10:MSC5 (S10M5 + OS) groups (Fig. 3).

MSCs cultured in media without OS differentiated into an osteoblast phenotype when in direct contact with calcified SMCs. A direct association was detected between the level of osteoblastic markers and the number of MSCs. By contrast, in the presence of MSCs, ALP activity $(\mathrm{P}<0.05)$ and ALP staining $(\mathrm{P}<0.05)$ were significantly higher in the calcified SMCs compared to the non-calcified SMCs in OS (Fig. 3). Moreover, the level of osteoblastic markers was dependent on the number of MSCs, with the level of osteoblastic differentiation of calcified SMC5: MSC10 (CS5M10) being higher compared to that of calcified SMC10: MSC5 (CS10M5) (Fig. 3B).

Wnt5a/Ror2 pathway may be associated with the differentiation fate of MSCs when in direct cell-cell contract with SMCs or calcified SMCs. Wnt5a protein is associated with the level of calcification, although it is rarely detected in noncalcification. Direct co-cultures were established by seeding together two different cell types at three distinct cell density ratios, culturing for 9 days, and examining the Wnt5a protein. Wnt5a protein was expressed in the groups CS15, CS5M10, CS10M5, with the highest expression evident in the CS5M10 group (Fig. 4). By contrast, no immunoreactivity for phosphotyrosine was detected in the groups S15, S10M5 + OS, while Wnt5a was slightly expressed in S5M10 + OS.

Ror 2 mRNA in non-calcified cells was higher than that in calcified cells. Ror 2 mRNA was quantitatively analyzed by real-time quantitative PCR. Ror2 mRNA in the groups with calcified SMCs was significantly reduced compared to that in groups S15, S5M10 + OS and S10M5 + OS (P<0.001) (Fig. 5). Compared to S10M5 + OS, Ror2 mRNA was expressed more 

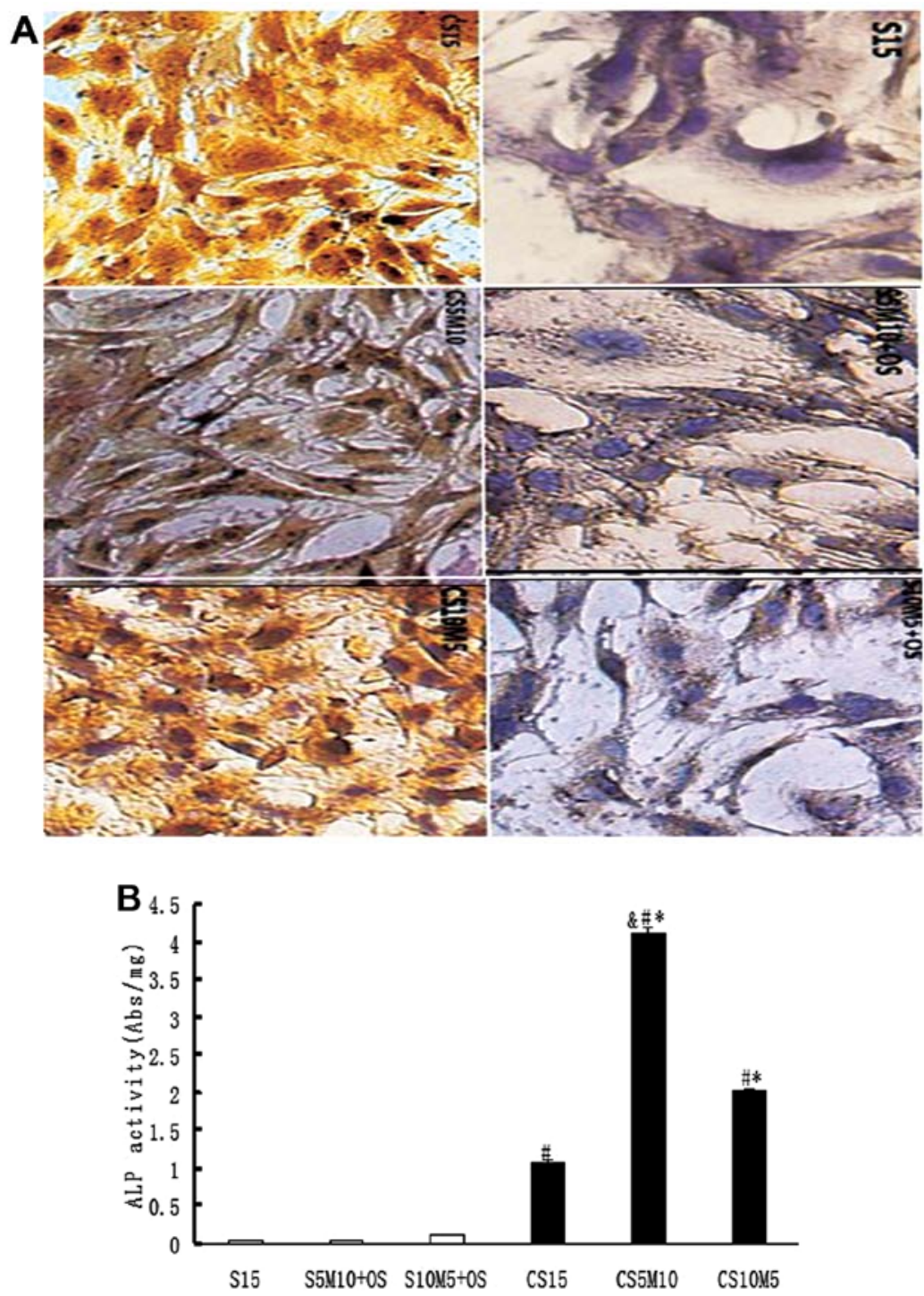

Figure 3. ALP staining (original magnification, $\mathrm{x} 400$ ) and ALP activity. Cells were seeded at a different cell density: SMCs or calcified SMCs $15 \times 10^{4}$; SMCs

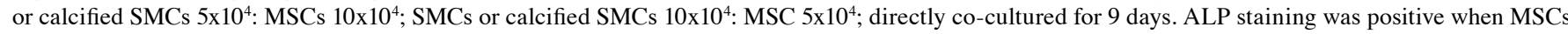
were directly co-cultured with calcified SMCs with a significant difference in the group of CS5M10, but negative when MSCs were directly co-cultured with non-calcified SMCs in culture medium with OS, with no significance detected when SMCs were cultured alone. ALP activity in the groups with calcified SMCs was higher compared to that in the groups with non-calcified SMCs $\left({ }^{*} \mathrm{P}<0.01\right)$, ALP activity was higher in the group when MSCs were directly co-cultured with calcified SMCs compared to calcified SMCs cultured alone (CS15) ("P<0.05). ALP activity in CS5M10 was higher than that in CS10M5 ( $\left.{ }^{*} \mathrm{P}<0.01\right)$.
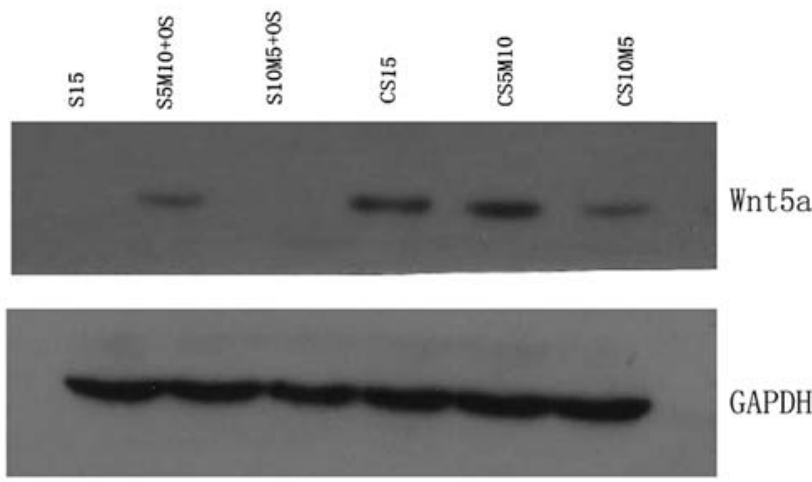

GAPDH

Figure 4. Western blotting shows expression of Wnt5a protein. Cells were seeded at a different cell density: SMCs or calcified SMCs $15 \times 10^{4}$; SMCs or calcified SMCs $5 \times 10^{4}$ : MSCs $10 \times 10^{4}$; SMCs or calcified SMCs $10 \times 10^{4}$ : MSCs $5 \times 10^{4}$; directly co-cultured for 9 days. Wnt5a protein was expressed in the groups with calcified SMCs with the highest expression in CS5M10. However, Wnt5a was only slightly expressed in S5M10 in groups with no-calcified SMCs.

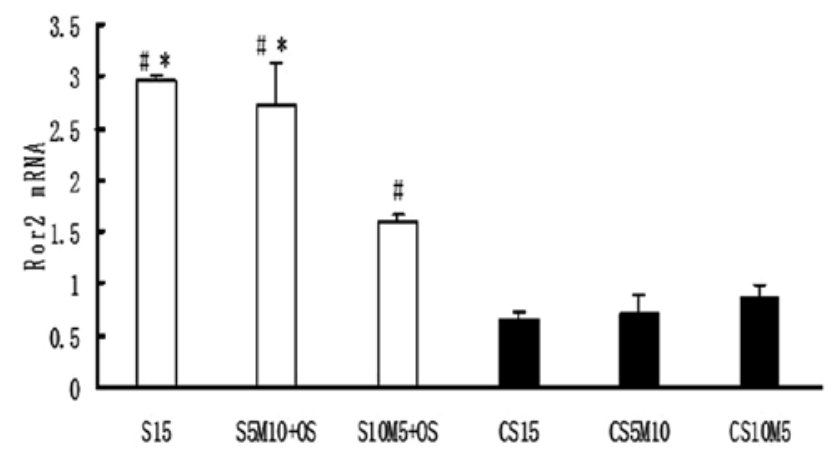

Figure 5. Ror2 mRNA expression. Cells were seeded at a different cell density: SMCs or calcified SMCs $15 \times 10^{4}$; SMCs or calcified SMCs $5 \times 10^{4}$ : MSCs $10 \times 10^{4}$; SMCs or calcified SMCs $10 \times 10^{4}$ : MSC $5 \times 10^{4}$; directly co-cultured for 9 days. Ror 2 mRNA in the groups with calcified SMCs was significantly reduced as compared to that in groups with non-calcified SMCs $\left({ }^{\#} \mathrm{P}<0.001\right)$. ${ }^{*} \mathrm{P}<0.05$ compared with S10M5 + OS. No difference was detected between CS15, CS5M10 and CS10M5. 
in groups $\mathrm{S} 15$ and $\mathrm{S} 10 \mathrm{M} 5+\mathrm{OS}(\mathrm{P}<0.05)$. Of note, there was no difference of Ror 2 mRNA expression between CS15, CS5M10 and CS10M5.

\section{Discussion}

MSCs play a critical role in tissue regeneration and homeostasis (20). However, findings of previous study showed that circulating concentrations of stem-cell-mobilizing cytokines were associated with the levels of osteoprogenitor cells and aortic calcification severity (21). Major factors, such us local environment and resident cells, are likely to determine the fate of MSCs $(8,22)$. A recent study has strongly suggested that cell-cell direct contact between resident cells and MSCs was critical in the differentiation of MSCs (12). Direct contact with distinct differentiated cells may be a critical determinant of mesenchymal stem cell fate in blood vessels and other connective tissues (8). To the best of our knowledge, this is the first study to examine whether MSCs should be co-cultured with non-calcified SMCs or calcified SMCs in vitro using a direct cell-cell co-culturing system. Direct cell contact affects the fate of MSCs (23). In this study, we found that MSCs did not differentiate into an osteoblast phenotype in the presence of non-calcified SMCs, whereas the spontaneous differentiation into bone-forming cells was observed when co-cultured with calcified SMCs, and dependent on the number of MSCs (Fig. 3).

However, the mechanism of action affecting this event remains to be determined. Many cell surface receptors, which have been used to create functional surfaces to enhance cell adhesion or alter cell morphology, have been identified, including cadherins (24) and cell adhesion molecules (CAM) (25). Wnt-signaling, which is mediated through cell-cell interaction and is involved in various developmental processes and cell functions (26), provides instructive cues for the recruitment, maintenance, and differentiation of MSCs (22). This signaling pathway on cell surface may be important in the differentiation of MSCs in direct cell-cell contact between MSCs and resident SMCs and calcified SMCs. It has been reported that canonical Wnt-signaling activates osteogenesis in mineralization under certain cellular contexts (27), and promotes the osteoblastogenesis of murine pluripotent mesenchymal and osteoprogenitor cells through the upregulation of RUNX2 (28) or osterix (29). However, canonical Wnt-signaling can be inhibited by the non-canonical Wnt-signaling pathway Wnt5a/Ror2 (16). Non-canonical signaling in general transduces through the c-Jun-N-terminal kinase $(\mathrm{JNK})$ /planar cell polarity or the calcium-releasing pathways and regulates cell movement $(30,31)$. Wnt5a, a member of the Wnt family is suggested to be involved in hydrophobic cell-cell interactions and is predominantly characterized as a non-canonical Wnt ligand that activates intracellular signaling via distinct receptors or co-receptors (31). Ror2, an orphan tyrosine kinase possessing an extracellular cysteine-rich Wnt-binding domain, has been shown to be a receptor for Wnt5a, inducing a non-canonical cascade involving the activation of JNK and inhibition of canonical signaling $(30,32)$. The interaction between Ror 2 and Wnt5a in order to mediate the non-canonical Wnt signaling pathway has received great attention in recent years $(30,32)$.
In addition, developmental phenotypes exhibiting an absence of Ror2 and Wnt5a lead to dwarfism, shortened limbs, facial abnormalities, ventricular septal defects in the heart, and abnormalities in lung development (33). In a previous study, it was found that Wnt5a may be involved in the pathogenesis of atherosclerosis as compared to protection from it (33), and that the expression of Wnt5a mRNA correlates with severity of atherosclerotic lesions (21). In this study, we investigated Wnt5a/Ror2 signaling in each group and observed that Wnt5a protein was expressed in the groups CS15, CS5M10 and SC10M5 with the highest expression being identified in CS5M10. By contrast, no immunoreactivity for phosphotyrosine occurred in the groups S15, S10M5 + OS, while Wnt5a was slightly expressed in S5M10 + OS (Fig. 4). Wnt5a protein was found to correlate with the severity of calcification, i.e., the more ALP activity, the more Wnt5a protein. Wnt5a was lightly expressed in S5M10 + OS (Fig. 4), likely due to the more MSCs in S5M10 + OS compared to S15 and S10M5 + OS.

The absence of Ror2 leads to enhanced Wnt/ $\beta$-catenin signaling, specifically in cells that have lost Ror2 expression (34), indicating that the intracellular domain of Ror2 is required for functional Wnt5a/Ror2 signaling. Taken together, those studies have suggested that Wnt5a/Ror2 signaling may inhibit canonical Wnt signaling in vivo, suggesting that Ror2 is a potential therapeutic target for human disease (34). In this study, Ror2 mRNA in the groups with calcified SMCs was significantly reduced compared to that in groups S15, $\mathrm{S} 5 \mathrm{M} 10+\mathrm{OS}$ and S10M5 + OS $(\mathrm{P}<0.001)$. Compared to S10M5 + OS, a higher expression of Ror2 mRNA was observed in groups $\mathrm{S} 15$ and S10M5 + OS $(\mathrm{P}<0.05)$. However, no difference of Ror 2 mRNA expression was detected between CS15, CS5M10 and CS10M5 (Fig. 5). Results of the present study show that the higher Ror2 mRNA expression, the less ALP activity of cells, and therefore less calcification since Ror2 mRNA in the groups with calcified SMCs was significantly reduced compared to that in groups $\mathrm{S} 15, \mathrm{~S} 5 \mathrm{M} 10+\mathrm{OS}$ and S10M5 + OS $(\mathrm{P}<0.001)$. Compared to S10M5 + OS, a higher Ror2 expression of mRNA was detected in groups S15 and S10M5 + OS $(\mathrm{P}<0.05)$ (Fig. 5).

In summary, MSCs are able to differentiate into different cell phenotypes when in direct cell-cell contact with SMCs or calcified SMCs, depending on the number of MSCs when differentiated into calcified cell phenotype. Additionally Wnt5a/Ror2 signaling may be associated with determination of the differentiation fate of MSCs in this process. Wnt5a/ Ror2 signaling and their regulators allow for the development of novel therapeutic strategies to prevent and treat valve and vascular calcification. Future studies should therefore focus on investigating the effect of Wnt5a/Ror2 signaling pathway in vascular calcification and stem cell therapy.

\section{References}

1. Demer LL and Tintut Y: Vascular calcification: pathobiology of a multifaceted disease. Circulation 117: 2938-2948, 2008.

2. Tanimura A, McGregor DH and Anderson HC: Matrix vesicles in atherosclerotic calcification. Proc Soc Exp Biol Med 172: 173-177, 1983.

3. Tanimura A, McGregor DH and Anderson HC: Calcification in atherosclerosis. I. Human studies. J Exp Pathol 2: 261-273, 1986. 
4. Boström KI, Rajamannan NM and Towler DA: The regulation of valvular and vascular sclerosis by osteogenic morphogens. Circ Res 109: 564-577, 2011.

5. Torsney E and Xu Q: Resident vascular progenitor cells. J Mol Cell Cardiol 50: 304-311, 2011.

6. Hirschi KK and Goodell MA: Hematopoietic, vascular and cardiac fates of bone marrow-derived stem cells. Gene Ther 9: 648-652, 2002.

7. Farrington-Rock C, Crofts NJ, Doherty MJ, et al: Chondrogenic and adipogenic potential of microvascular pericytes. Circulation 110: 2226-2232, 2004

8. Ball SG, Shuttleworth AC and Kielty CM: Direct cell contact influences bone marrow mesenchymal stem cell fate. Int $\mathrm{J}$ Biochem Cell Biol 36: 714-727, 2004.

9. Dufourcq P, Descamps B, Tojais NF, et al: Secreted frizzledrelated protein-1 enhances mesenchymal stem cell function in angiogenesis and contributes to neovessel maturation. Stem Cells 26: 2991-3001, 2008.

10. Cheung $\mathrm{C}$ and Sinha S: Human embryonic stem cell-derived vascular smooth muscle cells in therapeutic neovascularisation. J Mol Cell Cardiol 51: 651-664, 2011.

11. Zhao Y, Liu YX, Xie SL, Deng BQ, Wang JF and Nie RQ: Increased expression of granulocyte colony stimulating factor mediates mesenchymal stem cells recruitment after vascular injury. Chin Med J 124: 4286-4292, 2011.

12. Roorda BD, Elst A, Boer TG, Kamps WA and de Bont ES: Mesenchymal stem cells contribute to tumor cell proliferation by direct cell-cell contact interactions. Cancer Invest 28: 526-534, 2010.

13. Abedin M, Tintut Y and Demer LL: Mesenchymal stem cells and the artery wall. Circ Res 95: 671-676, 2004.

14. Kirton JP, Crofts NJ, George SJ, Brennan K and Canfield AE Wnt/beta-catenin signaling stimulates chondrogenic and inhibits adipogenic differentiation of pericytes: potential relevance to vascular disease? Circ Res 101: 581-589, 2007.

15. Shao JS, Cheng SL, Pingsterhaus JM, Charlton-Kachigian N, Loewy AP and Towler DA: Msx2 promotes cardiovascular calcification by activating paracrine Wnt signals. J Clin Invest 115 : 1210-1220, 2005.

16. Ishitani T, Kishida S, Hyodo-Miura J, et al: The TAK1-NLK mitogen-activated protein kinase cascade functions in the Wnt-5a/ $\mathrm{Ca}(2+)$ pathway to antagonize Wnt/beta-catenin signaling. Mol Cell Biol 23: 131-139, 2003

17. Sun S, Guo Z, Xiao X, et al: Isolation of mouse marrow mesenchymal progenitors by a novel and reliable method. Stem Cells 21: 527-535, 2003.

18. Minguell JJ, Erices A and Conget P: Mesenchymal stem cells. Exp Biol Med 226: 507-520, 2001.

19. Leskela HV, Risteli J, Niskanen S, Koivunen J, Ivaska KK and Lehenkari P: Osteoblast recruitment from stem cells does not decrease by age at late adulthood. Biochem Biophys Res Commun 311: 1008-1013, 2003.
20. Pal SN, Clancy P and Golledge J: Circulating concentrations of stem-cell-mobilizing cytokines are associated with levels of osteoprogenitor cells and aortic calcification severity. Circ J 75: 1227-1234, 2011.

21. Baksh D and Tuan RS: Canonical and non-canonical Wnts differentially affect the development potential of primary isolate of human bone marrow mesenchymal stem cells. J Cell Physiol 212: 817-826, 2007.

22. Yip CY and Simmons CA: The aortic valve microenvironment and its role in calcific aortic valve disease. Cardiovasc Pathol 20: 177-182, 2011.

23. Rogers GJ, Hodgkin MN and Squires PE: E-cadherin and cell adhesion: a role in architecture and function in the pancreatic islet. Cell Physiol Biochem 20: 987-994, 2007.

24. Yue XS, Murakami Y, Tamai T, Nagaoka M, Cho CS, Ito Y and Akaike T: A fusion protein N-cadherin-Fc as an artificial extracellular matrixm surface for maintenance of stem cell features. Biomaterials 31: 5287-5296, 2010.

25. Cheng CW, Yeh JC, Fan TP, Smith SK and Charnock-Jones DS: Wnt5a-mediated non-canonical Wnt signalling regulates human endothelial cell proliferation and migration. Biochem Biophys Res Commun 365: 285-290, 2008.

26. Bennett CN, Longo KA, Wright WS, et al: Regulation of osteoblastogenesis and bone mass by Wnt10b. Proc Natl Acad Sci USA 102: 3324-3329, 2005

27. Gaur T, Lengner CJ, Hovhannisyan H, et al: Canonical WNT signaling promotes osteogenesis by directly stimulating Runx2 gene expression. J Biol Chem 280: 33132-33140, 2005.

28. Pohjolainen V, Taskinen P, Soini Y, et al: Noncollagenous bone matrix proteins as a part of calcific aortic valve disease regulation. Hum Pathol 39: 1695-1701, 2008.

29. Grumolato L, Liu G, Mong P, Mudbhary R, et al: Canonical and non-canonical Wnts use a common mechanism to activate completely unrelated coreceptors. Genes Dev 24: 2517-2530, 2010.

30. Qiu W, Chen L and Kassem M: Activation of non-canonical Wnt/ JNK pathway by Wnt3a is associated with differentiation fate determination of human bone marrow stromal (mesenchymal) stem cells. Biochem Biophys Res Commun 413: 98-104, 2011

31. Yuan Y, Niu CC, Deng G, Li ZQ, Pan J, Zhao C, Yang ZL and Si WK: The Wnt5a/Ror2 noncanonical signaling pathway inhibits canonical Wnt signaling in K562 cells. Int J Mol Med 27: 63-69, 2011.

32. Niessen K and Karsan A: Notch signaling in cardiac development. Circ Res 102: 1169-1181, 2008.

33. Bhatt PM, Lewi CJ, House DL, et al: Increased Wnt5a mRNA expression in advanced atherosclerotic lesions, and oxidized LDL treated human monocyte-derived macrophages. Open Circ Vasc J 5: 1-7, 2012

34. Mikels A, Minami Y and Nusse R: Ror2 receptor requires tyrosine kinase activity to mediate Wnt5A signaling. J Biol Chem 284: 30167-30176, 2009. 